\title{
A neuromorphic approach to auditory pattern recognition in cricket phonotaxis
}

\author{
Thomas Rost*, Harshawardhan Ramachandran ${ }^{\dagger}$, Martin Paul Nawrot*, Elisabetta Chicca ${ }^{\dagger}$ \\ * Neuroinformatik / Theoretical Neuroscience \\ Freie Universität Berlin and Bernstein Center for Computational Neuroscience Berlin \\ Berlin, Germany \\ Email: thomas.rost@bccn-berlin.de \\ $\dagger$ Cognitive Interaction Technology - Center of Excellence \\ Bielefeld University, Germany
}

\begin{abstract}
Developing neuromorphic computing paradigms that mimic nervous system function is an emerging field of research with high potential for technical applications. In the present study we take inspiration from the cricket auditory system and propose a biologically plausible neural network architecture that can explain how acoustic pattern recognition is achieved in the cricket central brain. Our circuit model combines two key features of neural processing dynamics: Spike Frequency Adaptation (SFA) and synaptic short term plasticity. We developed and extensively tested the model function in software simulations. Furthermore, the feasibility of an analogue VLSI implementation is demonstrated using a multi-neuron chip comprising Integrate-and-Fire (IF) neurons and adaptive synapses.
\end{abstract}

\section{INTRODUCTION}

Insects provide highly interesting model systems for neuromorphic computation. They have sophisticated sensory systems that sub-serve accurate perceptual capabilities, and they show a large repertoire of complex behaviours. Yet, insects have small brains with limited neuronal resources. This allows constructing biologically realistic computational models of sensory computation, learning and memory formation, decision making, and behavioural control at the level of spiking neural networks.

Recognition and evaluation of acoustic patterns is a key element of mating behaviour in many animals. Phonotaxis is particularly well studied in crickets [1]. In Grillus bimaculatus males produce calling songs of a characteristic envelope pattern. Females evaluate these songs to recognize conspecifc males and to rate the fitness of a potential mating partner [2]. The stereotyped song pattern can easily be mimicked by artificial songs (Fig. 1 A) [3]. Behavioural experiments that quantify the phonotactic behaviour of females under variation of the artificial song parameters have shown that the most important aspect is encoded in the temporal sequence of pulses and pauses on a short time scale. High phonotactic scores are typically achieved for Pulse Periods (PPs) of $\sim 40 \mathrm{~ms}$ [4], [5]. In the auditory system of Grillus bimaculatus, a single neuron (Ascending Neuron 1 (AN1)) relays the relevant peripheral receptor neuron input to the central brain where auditory pattern recognition is achieved by a small-sized network of $\sim 30-50$ Central Brain Neurons (BNCs). While the properties of auditory receptor neurons and of the AN1 are well studied [6], [7], little is known about the mechanisms underlying auditory pattern recognition and behavioural decision making in the central brain. However, intracellular electrophysiological recordings from BNC activity [7], [8] showed that individual neurons exhibit a response behaviour that can be characterized by low-pass, high-pass, and band-pass characteristics with an optimal pass band that match the behaviourally optimal PP around $\sim 40 \mathrm{~ms}$.

Here, we suggest a biologically motivated spiking neural network model that provides a plausible mechanistic explanation of how the central brain circuit recognizes and evaluates the auditory pattern of a conspecific calling song. We demonstrate that our model qualitatively and quantitatively reproduces the electrophysiological measurements from BNCs during stimulation with artificial calling songs, both in soft simulation and on the neuromorphic hardware.

\section{MODEL}

\section{A. Model architecture}

Our circuit model is outlined in Fig. $1 \mathrm{C}$ and captures the basic blueprint of the acoustic system in Grillus bimaculatus. The model consists of four stages:

Auditory input: The Auditory input stage is not explicitly modelled. The envelopes of the $4.5 \mathrm{kHz}$ courtship songs are fed into the AN1 model as current patterns parametrized by their pulse width, pause duration, chirp length and chirp pause length (see figure $1 \mathrm{~A}$ ). In the present study only the short time-scale is considered, following the paradigm in [9].

AN1: The AN1 is modelled as a leaky IF neuron with SFA. The firing rate and adaptation characteristics have been matched to neurophysiological data [6].

BNC1: Brain neurons receive input from AN1. The synapses implement a short term plasticity model for depression and potentiation.

$B N C 2$ : BNC2 receives input only from the $\mathrm{BNC} 1$ units. It combines the high-pass and low-pass responses and acts as a band-pass filter.

\section{B. Software simulation}

All simulations have been performed with leaky IF neurons with SFA [10] using the Brian simulator [11]. The envelopes of the song patterns were introduced into the model as current 
A

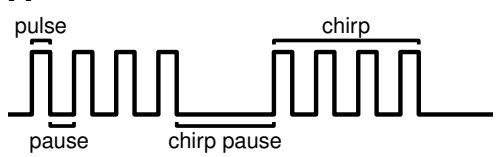

C

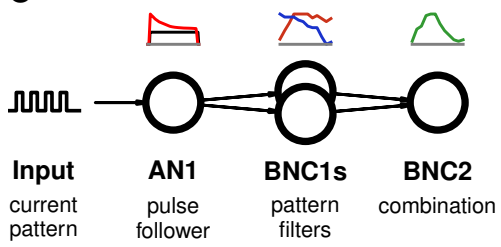

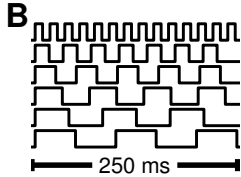

D

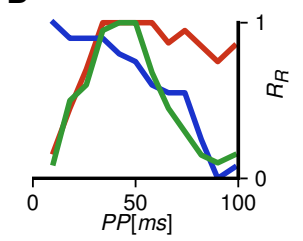

Fig. 1. Acoustic stimulus patterns and neural network model. A Temporal pattern of the calling song in Grillus bimaculatus. The carrier frequency of $4.5 \mathrm{kHz}$ is modulated by an envelope to produce the typical pattern, which is parametrized by the duration of pulses and pauses within chirps as well as the chirp and chirp pause duration. B Illustration of the stimulation paradigm used in [7] and [8]. Stimuli have a fixed duration of $250 \mathrm{~ms}$ and consist of pulses and pauses only, neglecting the natural chirp structure. The pulse duty cycle, i.e. the ratio of pulse duration and pause duration, is fixed while the PP is varied. C Neural network architecture. Model input to the AN1 follows the acoustic pulse pattern. The AN1 projects to BNC1 units, which act as lowpass or high-pass filters of the pulse pattern. A third stage (BNC2) integrates $\mathrm{BNC1}$ input and is tuned to reproduce the bandpass features. D Experimental filter curves for BNC neurons extracted from [9]. The relative response $\left(R_{R}\right)$ is plotted against the PP $(P P)$ of different stimulation patterns.

inputs into the AN1 unit. The pulse response of AN1 was matched to physiological data provided by Prof. Matthias Hennig's lab at the Humboldt University of Berlin. The firing rate and effective time-constant of adaptation were fitted following the method described in [12]. A small amount of variability was introduced by adding some filtered noise as an additional current input.

The BNC1 units receive input from AN1 through synapses which exhibit short-term plasticity. The model introduced by Tsodyks [13] was used to implement short-term facilitation and depression in these synaptic connections. Parameters for suitable filter properties were found via random sampling of the parameter space, allowing only pure facilitation or depression. Filter curves were obtained by summing spike counts over the stimulation window of $250 \mathrm{~ms}$ for different PP and then normalizing to the maximum response, matching the procedure in [7].

$\mathrm{BNC2}$ receives input from the BNC1 units. A genetic optimization algorithm was employed to find parameters which match the band-pass filter characteristics described by Schildberger [9].

\section{Hardware implementation}

A neuromorphic multi-neuron spiking chip and an Address Event Representation (AER) mapper [14] have been used for the hardware implementation of the network. The multi-neuron chip was fabricated using a standard AMS $0.35 \mu \mathrm{m}$ CMOS process and comprises a two-dimensional array of 32-by-64 leaky IF neurons [15]. Each neuron in the chip is connected to 3 synaptic circuits ( 2 excitatory, 1 inhibitory) each. The excitatory and inhibitory synapse circuits are based on the Differential-Pair Integrator (DPI) circuit proposed in [16].

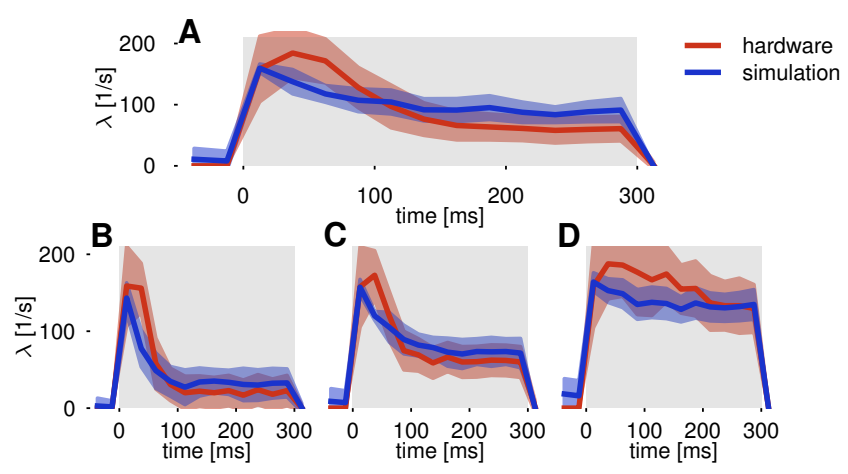

Fig. 2. Firing rates $(\lambda)$ of the two AN1 models stimulated with a step input. The gray region indicates the stimulation interval. Shadings represent standard deviations over 100 repeated trials for the simulation and over 100 units for the hardware model. A Parameters chosen for the AN1 model. B-D Illustration of different adaptation behaviours the simulation and hardware can produce.

These synapses produce Excitatory Post-Synaptic Currents (EPSCs) and Inhibitory Post-Synaptic Currents (IPSCs) with realistic temporal dynamics on the arrival of a pre-synaptic input spike. The AER mapper allows the implementation of a wide range of neural network topologies including multilayer networks and fully recurrent networks. The network topology is defined by programming a look-up table that the AER mapper reads to route spikes from source neurons to destination synapses.

We implemented SFA through an inhibitory synapse which feeds back to the pre-synaptic neuron. The inhibitory synapse produces an input negative current proportional to the output firing rate of the neuron [16]. To characterize the response variability in our hardware we stimulated a population of 100 leaky IF neurons with a $560 \mathrm{~Hz}$ input train of spikes lasting $300 \mathrm{~ms}$. We chose one neuron from this population as AN1 neuron feeding to the BNC1 neurons. The choice was made to match the simulation data in terms of peak firing rate and adaptation characteristics (time constant and steady state response).

The synaptic circuits receiving input spikes from the AN1 and connected to the BNC1 units were tuned to exhibit Short Term Potentiation (STP). This behavior is easy to achieve in the DPI synapse [16] but was surprising unexplored in neuromorphic systems so far. A weak synaptic weight and long time constant allows a slow build up of the postsynaptic current producing the STP effect. The BNC1 population consists of 100 leaky IF neurons, implemented with the same analog circuit used for the AN1 and using the same bias settings. The AN1 was stimulated with a train of spikes corresponding to the Schildbergers stimulation paradigm (see Fig. 1B).

\section{RESULTS}

A range of SFA characteristics can be obtained with both the software simulation and hardware (see Fig. 2). Stronger adaptation decreases the count variance across units on the hardware (figure $2 \mathrm{D}$ vs $\mathrm{B}$ ). The response properties of the AN1 model used to stimulate the Brain Neurons (BNs) are depicted in Fig. 2A. 


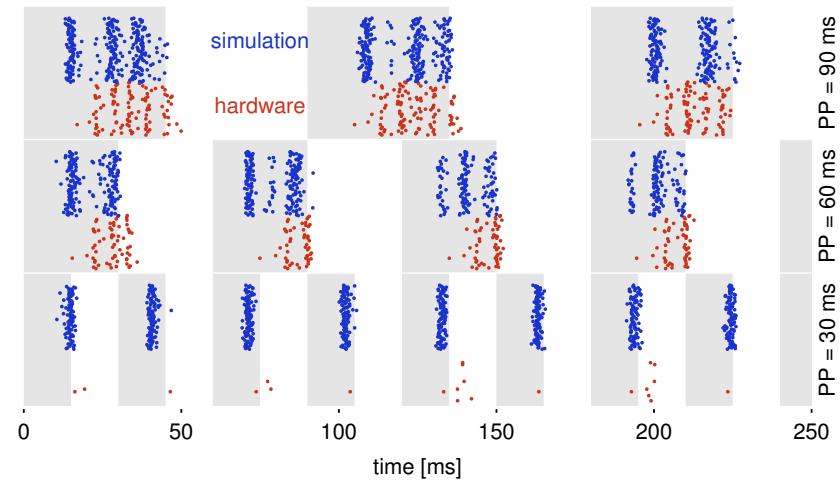

Fig. 3. Raster plot of a BNC1 low-pass filter implemented with STP for 100 units on the hardware and 100 trials in the simulation showing patterns with different PPs. The shaded regions indicate stimulation intervals. Due to the STP synapses, the units require a certain time of continuous stimulation to start spiking. Short pulse durations therefore evoke few spikes while longer pulses lead to bursting.

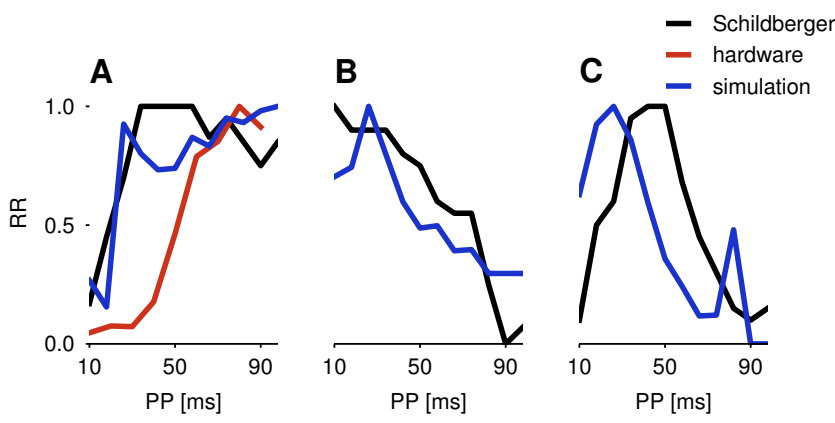

Fig. 4. Filter characteristics of BNC units for simulation and hardware. Superimposed are the filters from [9]. A BNC1-like low-pass filters using STP. On the hardware currently only STP is implemented. B BNC1-like high-pass filter using STD. C BNC2-like band-pass filter using STP.

Figure 3 illustrates the principle of pattern filtering using STP. In the rest state, synaptic weights are weak. It takes some time from the onset of a pulse to sufficiently increase the weights for the $\mathrm{BN}$ to produce a spike. As a consequence, the unit spikes very little for short pulses and produces burstlike patterns for longer stimuli. The unit is effectively a pulselength filter. This results in low-pass filtering as observed in [7], [17]. Equivalently, high-pass filters can be implemented using Short Term Depression (STD). Here, pulses cause the synaptic weights to be depressed and the unit only fires at the onset of pulses. Since the number of pulses in the stimulation interval decreases with PP in Schildberger's paradigm this effectively translates to high-pass filtering.

The high-pass and low-pass filters matching the ranges reported in [9] and [17] can be obtained using short-term synaptic plasticity with both hardware and software models. In this paper, we have shown only STP with hardware. The filter curves for the simulation have been calculated by averaging over 20 repeated stimulations with each stimulus pattern. For the hardware, the curve represents an average of $100 \mathrm{BNC} 1 \mathrm{~s}$ with identical parameters.

\section{DISCUSSION}

The neural network model presented here is based on anatomical and physiological evidence. The SFA in the AN1 is experimentally well documented and the major function of adaptation in this neuron is the generation of intensity invariance [6]. Moreover, SFA has been shown to reduce the response variability in sensory neurons, contributing to a reliable sound encoding [18].

The key functional aspect for the neural pattern recognition algorithm implemented here is short term plasticity which has been suggested in previous model studies [19], [20]. Other studies related to cricket phonotaxis have mainly focused on neurally inspired implementations of sensorimotor transformations for robotic applications [21], [22]. To date there is no experimental evidence for the existence of STD or STP synapses in the central cricket brain. However, short term plasticity has been found in insects, notably in the olfactory system of the fruit fly [23], [24].

For future studies it will be relevant to adapt the neural network model to natural sound patterns (as in Fig. 1A) in order to predict phonotactic scores available from large behavioural data sets [4]. Further, STD-filters will be implemented on the hardware. In addition, we plan to take into account neural processing of directional information, which is behaviourally relevant for localizing an attractive sound source.

\section{ACKNOWLEDGMENT}

This research is supported by the Excellence Cluster 227 (CITEC, Bielefeld University), the Collaborative Research Center Theoretical Biology (SFB 618), and the Bernstein Focus Neuronal Basis of Learning: Insect Inspired Robotics (grant 01GQ0941). T.R. receives a stipendship from the Graduate Training Center Sensory Computation in Neural Systems (GRK 1589). We thank Sadique Sheik, Fabio Stefanini and Emre Neftci for the python libraries used to operate the chips and their continuous support. We also thank Prof. Giacomo Indiveri for his valuable suggestions and ideas. Further we thank Prof. Matthias Hennig for experimental data and advice as well as Gundula Meckenhäuser for helpful discussions.

\section{REFERENCES}

[1] G. Pollack, "Who, what, where? recognition and localization of acoustic signals by insects," Current opinion in neurobiology, vol. 10, no. 6, pp. 763-767, 2000.

[2] B. Hedwig, "Pulses, patterns and paths: neurobiology of acoustic behaviour in crickets," Journal of Comparative Physiology A, vol. 192, no. 7, pp. 677-689, 2006.

[3] J. A. Doherty, "Trade-off phenomena in calling song recognition and phonotaxis in the cricket, gryllus bimaculatus (orthoptera, gryllidae)," Journal of Comparative Physiology A, vol. 156, no. 6, pp. 787-801, 1985.

[4] G. Meckenhäuser, R. M. Hennig, and M. P. Nawrot, "Critical song features for auditory pattern recognition in crickets," PloS one, vol. 8, no. 2, p. e55349, 2013.

[5] B. Grobe, M. M. Rothbart, A. Hanschke, and R. M. Hennig, "Auditory processing at two time scales by the cricket gryllus bimaculatus," The Journal of Experimental Biology, vol. 215, no. 10, pp. 1681-1690, 2012.

[6] J. Benda and R. M. Hennig, "Spike-frequency adaptation generates intensity invariance in a primary auditory interneuron," Journal of computational neuroscience, vol. 24, no. 2, pp. 113-136, 2008. 
[7] K. Schildberger, "Temporal selectivity of identified auditory neurons in the cricket brain," Journal of Comparative Physiology A, vol. 155, no. 2, pp. 171-185, 1984.

[8] K. Kostarakos and B. Hedwig, "Calling song recognition in female crickets: temporal tuning of identified brain neurons matches behavior," The Journal of Neuroscience, vol. 32, no. 28, pp. 9601-9612, 2012.

[9] K. Schildberger, "Temporal selectivity of identified auditory neurons in the cricket brain," Journal of Comparative Physiology A, vol. 155, no. 2, pp. 171-185, 1984.

[10] E. Muller, L. Buesing, J. Schemmel, and K. Meier, "Spike-frequency adapting neural ensembles: beyond mean adaptation and renewal theories." Neural computation, vol. 19, no. 11, pp. 2958-3010, Nov. 2007.

[11] D. F. M. Goodman and R. Brette, "Brian simulator," Scholarpedia, vol. 8, no. 1, p. 10883, 2013.

[12] J. Benda and R. M. Hennig, "Spike-frequency adaptation generates intensity invariance in a primary auditory interneuron." Journal of computational neuroscience, vol. 24, no. 2, pp. 113-36, Apr. 2008.

[13] M. Tsodyks, K. Pawelzik, and H. Markram, "Neural networks with dynamic synapses." Neural computation, vol. 10, no. 4, pp. 821-35, May 1998.

[14] D. Fasnacht and G. Indiveri, "A PCI based high-fanout AER mapper with $2 \mathrm{GiB}$ RAM look-up table, $0.8 \mu$ s latency and $66 \mathrm{mhz}$ output event-rate," in Conference on Information Sciences and Systems, CISS 2011, Johns Hopkins University, March 2011, pp. 1-6.

[15] G. Indiveri, E. Chicca, and R. Douglas, "A VLSI array of low-power spiking neurons and bistable synapses with spike-timing dependent plasticity," IEEE Transactions on Neural Networks, vol. 17, no. 1, pp. 211-221, Jan 2006.

[16] C. Bartolozzi and G. Indiveri, "Synaptic dynamics in analog VLSI," Neural Computation, vol. 19, no. 10, pp. 2581-2603, Oct 2007.

[17] K. Kostarakos, M. Hartbauer, and H. Römer, "Matched filters, mate choice and the evolution of sexually selected traits." PloS one, vol. 3, no. 8, p. e3005, Jan. 2008.

[18] F. Farkhooi, E. Muller, and M. P. Nawrot, "Adaptation reduces variability of the neuronal population code," Physical Review E, vol. 83, no. 5, p. 050905, 2011.

[19] R. Reeve, B. Webb, A. Horchler, G. Indiveri, and R. Quinn, "New technologies for testing a model of cricket phonotaxis on an outdoor robot," Robotics and Autonomous Systems, vol. 51, no. 1, pp. 41-54, 2005 .

[20] T. Rost, "Modelling pattern recognition in cricket phonotaxis," Master's thesis, Freie Universität Berlin, 2011, institutional Repository of the Freie Universität Berlin website. Available: http://edocs.fuberlin.de/docs/receive/FUDOCS_document_000000015361.

[21] B. Webb and T. Scutt, "A simple latency-dependent spiking-neuron model of cricket phonotaxis." Biological cybernetics, vol. 82, no. 3, pp. 247-69, Mar. 2000.

[22] P. Russo, B. Webb, R. Reeve, P. Arena, and L. Patan, "A cricket-inspired Neural Network For FeedForward Compensation and Multisensory Integration," in Proceedings of the 44th IEEE Conference on Decision and Control, and the European Control Conference. Seville, Spain, 12 $-15,2005$.

[23] H. Kazama and R. I. Wilson, "Homeostatic matching and nonlinear amplification at identified central synapses," Neuron, vol. 58, no. 3, pp. 401-413, 2008.

[24] H. Kazama and R. I. Wilson, "Origins of correlated activity in an olfactory circuit," Nature neuroscience, vol. 12, no. 9, pp. 1136-1144, 2009. 